\title{
-NOTES-
}

\section{NOTE ON THE TORSIONAL RIGIDITY OF SEMI-CIRCULAR BARS*}

By P. L. SHENG (Taipeh, Taiwan)

In such cases, the general stress function for torsion for the cross-section of a circular sector $^{1}$ reduces to

$$
\Psi=\frac{1}{2}\left[-r^{2}(1+\cos 2 \theta)+\frac{16 a^{3}}{\pi} \sum_{n=1,3,5}^{\infty}(-1)^{(n+1) / 2}\left(\frac{r}{a}\right)^{n} \frac{\cos n \theta}{n\left(n^{2}-4\right)}\right],
$$

where $r, \theta$ are the polar coordinates, and $a$ the radius of the semi-circle. It is easy to verify that $\Psi=0$ at the boundary.

The applied torque $T$ is given by the well-known formula

$$
T=2 G \alpha \int_{A} \Psi d A=2 G \alpha \iint_{A} \Psi r d r d \theta,
$$

where $\int_{A}$ or $\iint_{A}$ are taken over the cross-sectional area, $G$ denotes the shearing modulus of elasticity, and $\alpha$ the angle of twist per unit length of the bar.

Hence

$$
\begin{aligned}
& T=G \alpha\left[-\int_{-\pi / 2}^{\pi / 2} \int_{0}^{a} r^{3}(1+\cos 2 \theta) d r d \theta\right. \\
& \left.+\frac{16 a^{3}}{\pi} \sum_{n=1,3,5, \ldots}^{\infty} \frac{(-1)^{(n+1) / 2}}{n\left(n^{2}-4\right)} \int_{-\pi / 2}^{\pi / 2} \int_{0}^{a}\left(\frac{r}{a}\right)^{n+1} \cos n \theta d r d \theta\right] \\
& =G \alpha\left[-\frac{\pi}{4} a^{4}-\frac{32 a^{4}}{\pi} \sum_{n=1,3,5, \ldots}^{\infty} \frac{1}{(n-2) n^{2}(n+2)^{2}}\right] .
\end{aligned}
$$

Using the well-known formula

$$
\sum_{n=1,3,5, \ldots}^{\infty} \frac{1}{n^{2}}=\frac{\pi^{2}}{8}
$$

we can easily derive by the split

$$
\frac{1}{(n-2) n^{2}(n+2)^{2}}=\frac{1}{32}\left[\frac{1}{(n-2) n}+\frac{5}{n(n+2)}-\frac{4}{n^{2}}-\frac{2}{(n+2)^{2}}\right]
$$

that

$$
\sum_{n=1,3,5, \ldots}^{\infty} \frac{1}{(n-2) n^{2}(n+2)^{2}}=\frac{1}{8}\left[1-\frac{3}{16} \pi^{2}\right]
$$

*Received September 30, 1950.

${ }^{1}$ Cf., e.g., S. Timoshenko's Theory of elasticity, $\$ 79$, noticing that in Timoshenko's book the stress function $\phi$ is $\phi=G \alpha \Psi$, and $T, \alpha$, and $(r, \theta)$ in the present paper are written as $M_{t}, \theta$, and $(r, \psi)$ respectively. 
By substitution,

$$
T=G \alpha a^{4}\left[\frac{\pi}{2}-\frac{4}{\pi}\right]
$$

exactly, and the numerical expression is

$$
T=0.297,556,782 G \alpha a^{4}
$$

which differ slightly from St. Venant's result of 0.296 or $0.2966 .^{2}$

2The value 0.296 is from the table in S. Timoshenko's Theory of elasticity, p. 250, 1st ed., 2nd and 8th impression (1933 and 1934), McGraw-Hill; while from the datum given in I. Todhunter and K. Pearson's $A$ history of the theory of elasticity and of the strength of materials, vol. II, part I, p. 193, we have

$$
T=0.3776 M=0.3776 \times G \alpha \times\left(\pi a^{2}\right) / 2 \times a^{2} / 2=0.2966 G \alpha a^{4} .
$$

A NOTE ON MY PAPER

\section{ON STEADY LAMINAR TWO-DIMENSIONAL JETS IN COMPRESSIBLE VISCOUS GASES FAR BEHIND THE SLIT*}

Quarterly of Applied Mathematics, 7, 313-323 (1949)

BY M. Z. KRZYWOBLOCKI (University of Illinois)

Determination of the constant of integration for the temperature distribution (p. 317, eq. 25) from the condition that that total flux of enthalpy across jet is alike at all cross-sections restricts solution to small Mach numbers (if the comparison cross-section is close to the slit, as pointed out by A. H. Shapiro, Appl. Mech. Rev. III (1950) p. 415, No. 2718) or to high Mach numbers (if the comparison section is far from the slit). To take into account all the relative cases, that constant may be determined from the condition that the total flux (enthalpy plus kinetic energy) is alike at all cross-sections:

$$
2 \int_{0}^{\infty}\left(J c_{p} T_{1}+u_{1}^{2} / 2\right) u_{1}\left(\rho_{\infty}+\rho_{1}\right) d y=\text { const. }
$$

${ }^{*}$ Received Feb. 22, 1951.

\section{ON THE LEAST EIGENVALUE OF HILL'S EQUATION*}

By C. R. PUTNAM (The Institute for Advanced Study, Princeton)

The differential equation

$$
x^{\prime \prime}+[\lambda+f(t)] x=0,
$$

in which $\lambda$ is a real parameter and $f(t)$, for $-\infty<t<\infty$, is a real-valued, continuous, periodic function $(\not \equiv 0)$, arises in problems dealing with the propagation of waves in

*Received November 10, 1950. 